Reflexiones contables (Cúcuta)

20

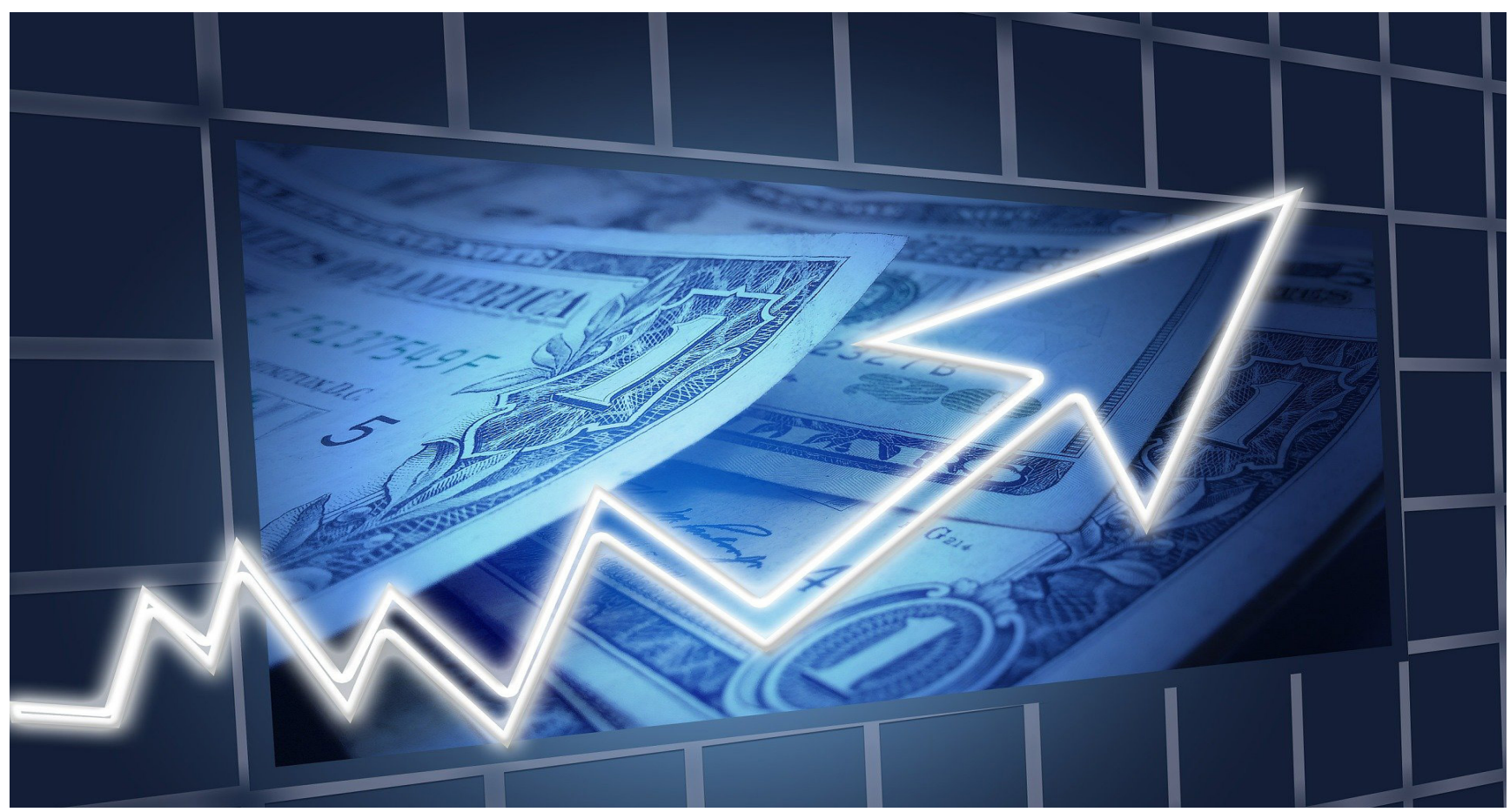

\title{
El regreso de Uber en la economía de la ciudad de Cúcuta
}

\section{Uber's return in the city's economy of Cúcuta}

Diana Alejandra Delgado-Moreno

Estudiante de Administración Financiera. Fundación de Estudios

Superiores de Comfanorte. Correo: est_da.delgado@fesc.edu.co
William Oswaldo Berbesí-Carrillo

Estudiante de Administración de Negocios Internacionales. Fundación de Estudios Superiores de Comfanorte. Correo: est_wo_ berbesi@fesc.edu.co 
Cómo citar: Delgado-Moreno, D.A., Berbesí-Carrillo, W.O. (2020). El regreso de Uber en la economía de la ciudad de Cúcuta. Reflexiones contables (Cúcuta), 3(2), 20-28.

\section{Resumen}

El objetivo principal de ésta investigación fue analizary determinar la incidencia que ha tenido plataformas digitales de servicio vehicular, en este caso, UBER en la economía de la ciudad de Cúcuta, debido a que con su retiro muchos habitantes de la ciudad se quedaron sin empleo. Se analizaron las estadísticas de desempleo en la ciudad de Cúcuta, junto con la encuesta establecida para determinar la percepción de los usuarios y prestadores de servicio de UBER con respecto a su regreso al país. Dicha encuesta se realizó en la ciudad de Cúcuta. La información recolectada por la encuesta y las estadísticas del DANE y la Cámara de Comercio, demostraron que Cúcuta basa su economía por medio de la informalidad, siendo así que plataformas digitales de servicio vehicular, terminan incidiendo de manera directa en la economía de la ciudad.

Palabras clave: Economía, Incidencia, Informalidad, Uber.

\section{Abstract}

The main objective of this research was to analyze and determine the impact that digital platforms of vehicular service, in this case, UBER, have had on the economy of the city of Cúcuta, due to the fact that with its withdrawal many inhabitants of the city were left without employment. Unemployment statistics in the city of Cúcuta were analyzed, together with the survey established to determine the perception of UBER users and service providers regarding their return to the country. This survey was conducted in the city of Cúcuta. The information collected by the survey and the statistics of DANE and the Chamber of Commerce, showed that Cúcuta bases its economy through informality, being so that digital platforms of vehicle service, end up directly impacting the economy of the city.

Keywords: Economy, Incident, Informality, Uber. 


\section{2}

\section{Introducción}

En la actualidad, la ciudad de Cúcuta posee un $70 \%$ de trabajo informal, el cual ha beneficiado enormemente a la economía de la misma. La llegada de plataformas como UBER, In Driver, o Cabify, ha generado un nuevo tipo de empleo para los ciudadanos; sin embargo, es de destacar que este tipo de trabajo, no ha sido oficializado, por lo que aún se consideraría informal. El pasado 31 de enero, la plataforma UBER fue obligada a salir del país, debido a un decreto presentado en diciembre del 2019, por una tutela impuesta por ASOTAXIS, quienes ven éste servicio como su perdición. Su salida, dejó a más de 600 cucuteños, que operaban como conductores de la plataforma UBER, en el desempleo. El 20 de Febrero del 2020, UBER volvió a Colombia, presentando un mejorado servicio tanto para trabajadores, como clientes. (Narváez 2018).

Con lo anterior mencionado se ha llegado a ver la fuerte competencia que tiene UBER sobre los servicios de transportes cotidianos como lo son los TAXIS, ya que son los principalmente afectados gracias a que los usuarios declaran que en servicio al cliente, amabilidad y eficacia es más óptimo el servicio de UBER a partir de fuertes declaraciones frente al mal servicio y pésimo trato de los conductores de TAXIS hacía sus pasajeros.

La razón por la cual se hizo esta investigación, es para conocer la incidencia que tiene la plataforma UBER dentro de la economía de Cúcuta, dado que un gran afluente de personas presta sus servicios en éste nuevo tipo de negocio. Se ha investigado estadísticas y percepción del usuario para poder establecer los parámetros de incidencia en la economía de la ciudad. La actual investigación plantea ser un precedente para futuras investigaciones de incidencia económica de plataformas digitales en la ciudad de Cúcuta, debido a que anteriormente no se tenía artículos relacionados a ésta temática. (Ossa 2017).

\section{Bases teóricas}

Para poder entender las dinámicas del retorno de UBER a Colombia y su incidencia dentro del marco comercial y económico dentro de la ciudad de Cúcuta, es importante tener en cuenta ciertos aspectos que permitirán obtener un análisis más amplio acerca de la investigación.

\section{Desempleo en Cúcuta}

El desempleo sigue siendo uno de los principales problemas a nivel nacional y es claro que dada la situación actual, sea peor. La tasa de desocupación nacional en marzo del 2020, presentó un aumento de 1,8 p.p con respecto al año anterior, ubicándose en $12,6 \%$. Cúcuta por su parte, representaría un tercer lugar a nivel nacional con un $19,8 \%$, una cifra que superó incluso la dada en el 2018 (de 19,5\%), pero que se evidencia más esto con respecto al año 2019 con un 17,4\% (Figura 1). Lo cual indica que Cúcuta y su área metropolitana, sigue teniendo los más altos índices de desempleo en el país. Comercio, reparación de autos, manufactureras fueron los sectores que presentaron los índices más altos de ocupación, sin embargo, siguen siendo bajos números a comparación de la informalidad presentada en la ciudad. (Coronel 2005). 
HISTORICO TASA DE DESEMPLEO (\%)

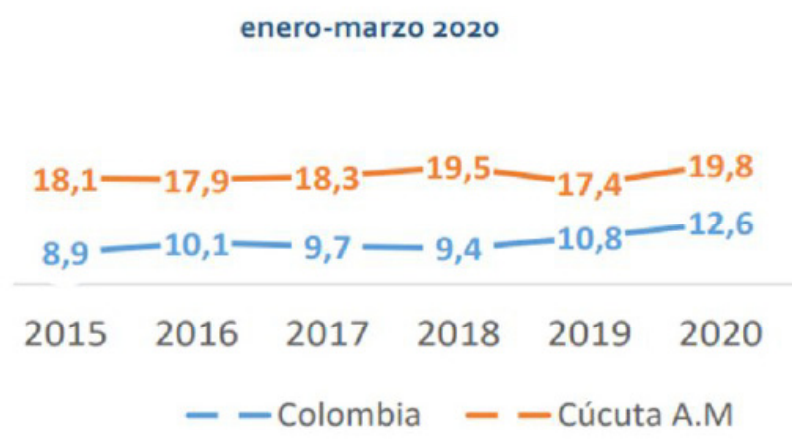

Imagen 1. Historico del desempleo

Fuente: Cámara comercio Cúcuta 2020

Las cifras son preocupantes, pero claras al momento de entender que la ciudad afronta actualmente uno de los picos más alto de desempleo que se han visto en la región. Una razón de esto está dado por el cierre de fronteras y el aumento de informalidad en la ciudad de Cúcuta. Aun siendo frontera, el cierre de ésta genera grandes pérdidas en la ciudad y en la región.

\section{Tasa de informalidad en la ciudad}

Cúcuta cuenta con la tasa más alta de informalidad del país, llevando un histórico $71,4 \%$, debido a que las garantías de los trabajos formales, no son suficientes para establecerse en uno. Es por ello, que el cucuteño promedio se adecúa a estos trabajos que de cierta manera, no poseen las herramientas necesarias para garantizar su seguridad, sin embargo, es la única opción que le queda para obtener sustento. La llegada de UBER a Colombia, permitió otro tipo de trabajo de informalidad, el cual permitió a más de 600 cucuteños, trabajar dentro de la plataforma.

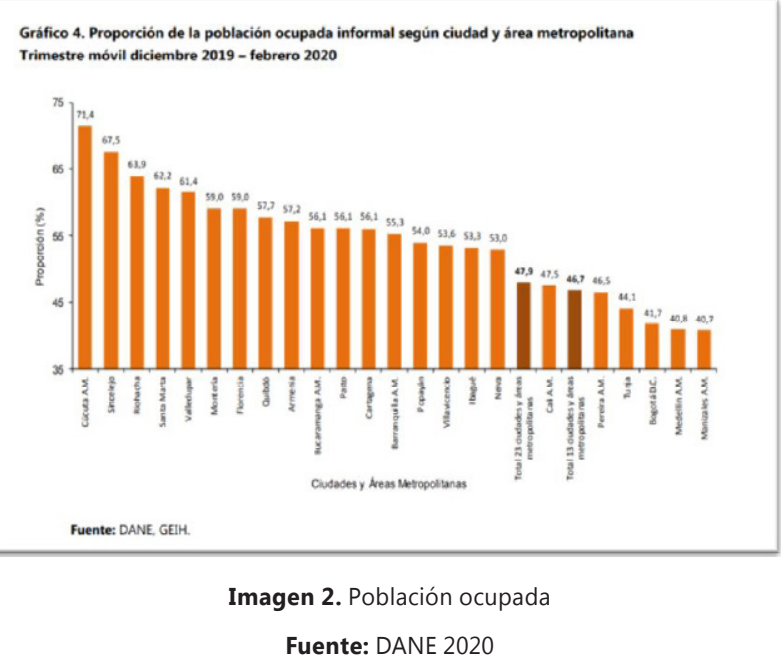

Cúcuta volvió a ser la ciudad con más informalidad laboral. Los datos revelan que de los 10.000 nuevos ocupados en 2019, solo 1.000 entraron al mercado formal. Esto quiere decir que en total 9,000 desempleos se aumentaron a la cifra en la ciudad, generando preocupación en la economía. (Avalos 2015). "En la ciudad hubo una reactivación de la generación de empleo en el sector manufacturero, en el caso del calzado, los plásticos y la confección", dijo Juan Daniel Oviedo, director del DANE. Sin embargo, esto solo quiere decir que son esos sectores los que han permitido los 1000 empleos formales de los 10,000 ocupados. (Castro 2016).

\section{Nuevas estrategias de UBER}

UBER se replanteó para poder volver con las medidas que considera necesarias para establecerse dentro del marco económico de la ciudad. Es por eso, que aplicó nuevas estrategias con el fin de garantizar un mejor servicio. Entre las nuevas medidas que empezó a poner en marcha la plataforma de UBER (Chavez 2016). y lo innovador, es el hecho de alquilar un vehículo con conductor, esto significa que se hace un contrato tanto con el usuario como con el prestador del servicio. (Hernández 2018), Para el que trabaja con la plataforma, puede colocar su 


\section{4}

carro a disposición de la empresa y éste le hace firmar un contrato por parte y parte donde se le asegura que su auto tendrá las medidas necesarias de control. Ahora, por parte del usuario, deberá "firmar" o aceptar un contrato en el momento en que pida el servicio, es decir, con un clic adicional, acepta los términos y condiciones ofrecidos por la plataforma y el arrendatario del auto.(Monsalve 2019).

Dicho contrato durará lo que dure el viaje asignado o deseado. Una vez finalizado, terminará el contrato de ése usuario y volverá a repetirse el mismo proceso con el siguiente. En un correo electrónico enviado a sus usuarios, la empresa informa que cada contrato cuenta con un seguro que cubre "responsabilidad civil" y "accidentes personales". Adicional, ofrece los siguientes servicios:

- Por Horas: El cliente puede alquilar un vehículo con conductor y pagarlo por el tiempo en que desee.

- Uber YA: Se alquilará un vehículo con conductor para poder moverse de manera más rápida.

- Economy: Se alquilan vehículos con conductor, pero se busca modelos de autos no tan recientes para minimizar los costos.

- Comfort: La alternativa Premium permite alquilar vehículos más modernos con conductor.

- XL: Ideal para viajes en grupo. El precio del arrendamiento del vehículo, se puede dividir entre todos los que hagan parte del viaje. (Castro 2015).

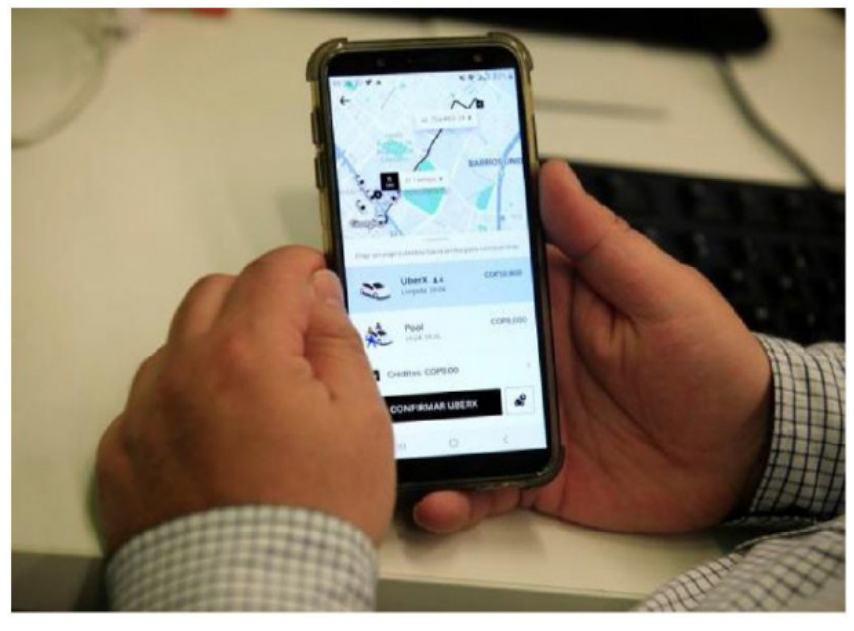

Imagen 3. arrendamiento de vehículos Fuente: (Tiempo 2020)

Para los usuarios de UBER, el retiro de la plataforma afectó no solo la economía de la ciudad, sino también obligó a aquellos usuarios a volver a un sistema que para ellos, presenta muchas falencias, como es el gremio de Taxis. Si bien, las nuevas medidas impuestas por UBER pueden cambiar el hecho de que hayan más trabajadores, es necesario entender que la ciudad ya se adecuó a éste nuevo negocio. Para comprender mejor todo esto, se dispone en ésta investigación de una encuesta realizada a usuarios y prestadores del servicio, donde se visualiza la percepción que tienen estos del regreso de UBER a Colombia y su incidencia en la ciudad. (Lesmes 2020).

Actualmente el país atraviesa una pandemia mundial, por lo que incluso servicios como UBER han tenido que frenar sus actividades laborales completamente durante la emergencia; sin embargo, el 15 de mayo del 2020 anunció que reestablecerá sus funciones a la normalidad. Todo esto, bajo las medidas necesarias de seguridad decretados por el Gobierno Nacional. (Abascal 2009). El conductor que preste el servicio debe certificar que está autorizado para salir, es decir, debe contar con los permisos respectivos por parte de las autoridades para prestar esta opción de movilidad. (Carballo 2017). El conductor también debe probar que está usando un 
tapabocas a través de una fotografía, que debe compartir con Uber para que valide el servicio. El pasajero también deberá hacer un chequeo de que los protocolos establecidos estén siendo respetados, como la limpieza del auto, como el lavado de manos. En cada vehículo pueden ir como máximo dos pasajeros, con tapabocas, que deben desinfectarse con gel antibacterial antes y después de subirse al vehículo. Esto garantiza que se brinde un buen servicio y que adicional, se pueda seguir operando con total normalidad aún en ésta emergencia mundial. (Castro et al 2018).

\section{Materiales y métodos.}

Para ésta investigación se planteó el uso de datos cuantitativos, debido a que se usó al DANE y a la Cámara de Comercio para establecer la información pertinente en cuanto a los índices de desempleo y tasa de informalidad en la ciudad de Cúcuta. Además, se usó la encuesta para obtener los datos cualitativos, que corresponden a la percepción del cliente con referente al producto-servicio que presta UBER. Se realizó ésta herramienta de recolección de datos de un total de 22 encuestados, entre los que se encuentran usuarios y prestadores de servicio dentro de la plataforma UBER.

\section{Resultados y análisis}

Se realizó una encuesta a 22 personas para conocer la percepción que tienen de la plataforma UBER y se analizaron los resultados. La encuesta está dirigida a usuarios y a prestadores del servicio de UBER.
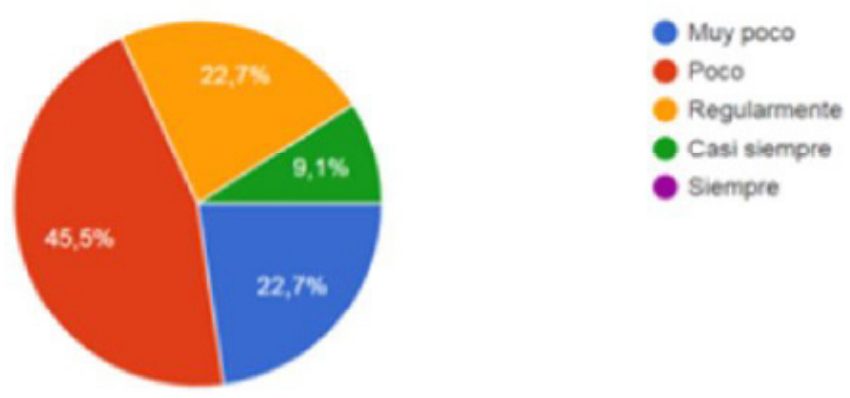

Imagen 4. ¿Con que frecuencia usa los servicios de Uber?

Fuente: los autores.

Se puede observar en la imagen 4 que aun cuando la mayoría de los encuestados usa poco o muy poco el servicio, igual lo han usado, siendo así que se determina la participación de ésta plataforma por parte de los usuarios en la ciudad de Cúcuta. Un muy poco abarcó un $22,7 \%$ (5 votos), Poco un 45,5\% (10 votos), Regular $22,7 \%$ (5 votos) y Casi siempre con un $9,1 \%$ (2 votos).

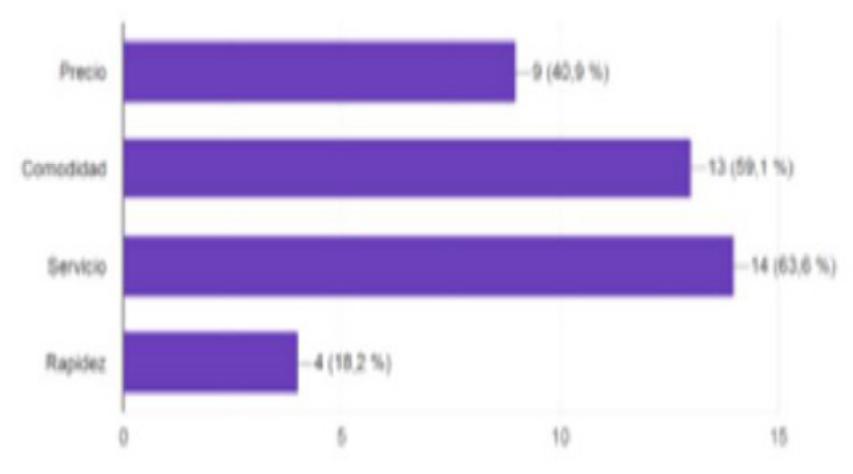

Imagen 5. ¿Cuales son los factores más llamativos de Uber?

Fuente: los autores.

Para llegar a conocer mejor la percepción del usuario y del prestador del servicio dentro de la plataforma, se determinaron los factores más importantes que ven. Para ésta pregunta se dejó la opción de múltiple respuesta para que se tuviese un estándar de respuestas varias. Los resultados muestran que un 63,6\% (14 votos) consideran que es más llamativo el Servicio que ofrece UBER, mientras que la Comodidad tuvo un 59,1\% (13 votos), Precio $40,9 \%$ (9 votos) y Rapidez un 18,2\% (4 votos). 


\section{6}

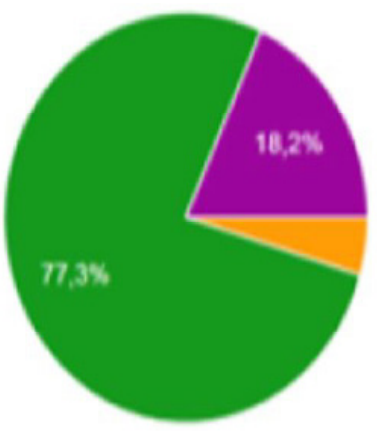

Imagen 6. El trato que se ofrece a los clientes es:

Fuente: los autores

Confirmando lo anterior, los usuarios del servicio de UBER sienten que la atención y el trato que se les ofrece son, en su mayoría, buenos. La percepción de Bueno, está con un $77,3 \%$ (17 votos), Muy bueno un 18,2\% (4 votos) y Regular un 4,5\% (1 voto).

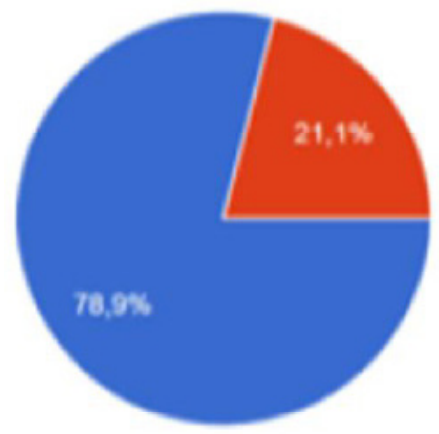

Imagen 7. Si usted presta sus servicios dentro de la plataforma Uber, ¿considera que las nuevas medidas implementadas por la empresa son adecuadas?

Fuente: los autores

Ésta pregunta iba dirigida principalmente a prestadores de servicio en la plataforma UBER, por lo que era la única no obligatoria de responder en la encuesta. Con un $78,9 \%$ (15 votos), aquellos que prestan sus servicios dentro de la plataforma se sienten conformes con las nuevas estrategias que desea implementar UBER. Mientras que con un $21,1 \%$ (4 votos), estos mismos no se sienten satisfechos o no están de acuerdo a las medidas que la plataforma está implementando.

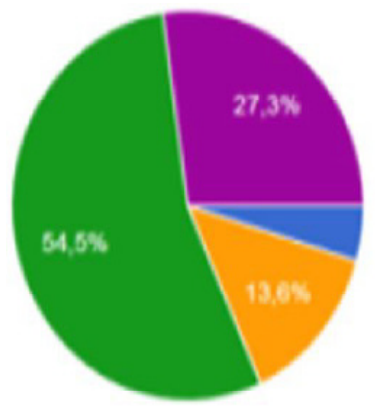

Nada de acuerdo

Poco de acuerdo

Indiferente

De acuerdo

Muy de acuerdo

Imagen 8. ¿Esta de acuerdo con el ingreso de Uber a Colombia?

Fuente: los autores

Es importante conocer la idea que tiene el consumidor con respecto al regreso de UBER a Colombia, así que según la encuesta, el usuario se siente muy de acuerdo de que la plataforma haya vuelto a funcionar dentro del territorio nacional y aún más, dentro de la ciudad. Cúcuta ha venido sufriendo los cambios en la economía del país y la llegada de éstas plataformas generaron un nuevo tipo de empleo informal que de por sí, contribuye en mayor medida con la economía de la ciudad. Un Muy de acuerdo con un 27,3\% (6 votos), un De acuerdo se ratifica con un $54,5 \%$ de las respuestas (12 votos). Por otro lado, una minoría como tal, se encuentran Indiferentes con un $13,6 \%$ ( 3 votos) y Nada de acuerdo con un $4,5 \%$ (1 voto).

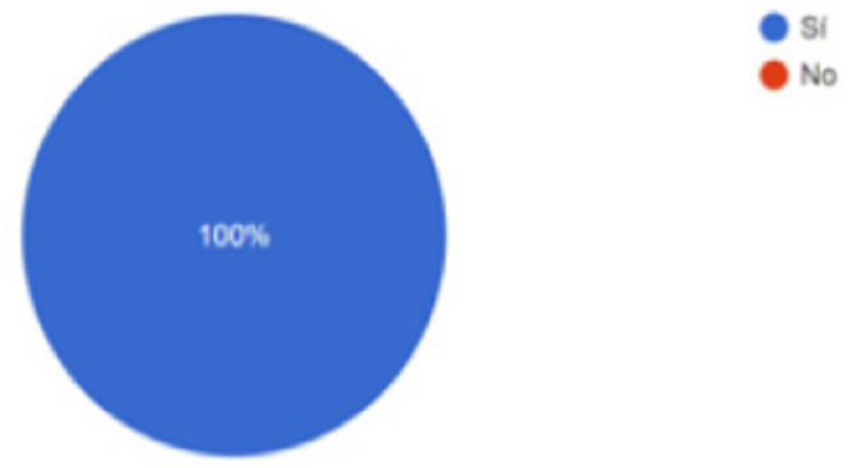

Imagen 9. ¿Considera usted que las plataformas como Uber, benefician la economía de la ciudad de Cúcuta

Fuente: los autores

Con una absoluta respuesta, tanto de usuarios como aquellos que prestan sus servicios dentro de la plataforma, la ciudad se ve beneficiada en gran medida con el retorno de UBER a 
Colombia. Si bien es de entender que no solo da un respiro a aquellos que creían haberlo perdido todo cuando la plataforma se fue en Febrero, sino que también le genera a la ciudad un cambio positivo en materia económica, beneficiando así el mayor mercado que se maneja en Cúcuta, que es la informalidad.

\section{Conclusiones}

La información recolectada por la encuesta y las estadísticas del DANE y la Cámara de Comercio, demostraron que Cúcuta basa su economía por medio de la informalidad, siendo así que plataformas digitales de servicio vehicular, terminan incidiendo de manera directa en la economía de la ciudad. UBER durante sus 20 días fuera del servicio en el país, afectó de manera considerable a los más de 600 cucuteños que prestaban sus servicios en la plataforma. Si bien regresó el pasado 20 de Febrero, lo hizo renovando sus servicios, lo cual tiene cierta incertidumbre de lo que puede llegar a ser éste cambio en un futuro, debido a que, como se demostró en la encuesta realizada a usuarios y prestadores del servicio, no todos están de acuerdo con las nuevas medidas que la empresa adoptó; sin embargo, algo es seguro y es que terminará teniendo un impacto en la economía local otra vez.

\section{Referencias}

Abascal, Arturo (2009), "El servicio público de taxi”, en Régimen Jurídico del Urbanismo. Memoria del Primer Congreso de Derecho Administrativo Mexicana. Fernández Ruíz, Jorge; Germán Cisneros Farías y Filiberto Otelo Salas (coord.), México, unam, pp. 225-257.

Ávalos, Marcos y Paula Sofía (2015), "Baby, you can ('t) drive my car. El caso de Uber en México", en Economía informa, núm. 390, enero-febrero 2015, pp. 104-112.

\section{7}

Castro, Miriam (2015), “Traxi: una app para saber si tu taxi es confiable", en Milenio. com, 15 de junio, México, en http://www. milenio.com/negocios/emprendedores/ ubertaxis_cdmx-taxistas_df-usuarios_ubertraxicabify_0_536946489.html, consultado el 1 de julio de 2015.

Chávez, Orlando (2016), “UBER llega como una alternativa de transporte", en El Diario, lealtad a Chihuahua, en http:// eldiariodechihuahua. mx/Estado/2016/06/21/ desplaza-a-3-milviolencia-en-sinaloa/, consultado el 22 de julio de 2016.

cnn Expansión (12/06/2015), “Las ciudades que le han cerrado las puertas a Uber", México, en http://www.cnnexpansion.com/ tecnologia/2015/06/10/las-controversiasdeuber, consultado el 20 de junio de 2015

Castro Correa, J. A., Sepúlveda Mora, S. B., Medina Delgado, B., \& Guevara Ibarra, D. (2018). Servicio web para la geolocalización de los vehículos de transporte público en la ciudad de Cúcuta. Respuestas, 23(S1), 29-37. https://doi.org/10.22463/0122820X.1498

Castro Alfaro, A. (2016). Estrategias para la disminución de la carga impositiva en las organizaciones empresariales. Enfoque Disciplinario, 1(1), 21-34. Recuperado a partir de http:/enfoquedisciplinario.org/revista/ index.php/enfoque/article/view/6

Carballo Mass, E. (2017). Modelo de negocio para una tienda virtual de venta de llantas al sector público mediante la modalidad de contratación. Conocimiento Global, 2(1), 20-40. Recuperado a partir de http:// conocimientoglobal.org/revista/index.php/ cglobal/article/view/14

Coronel López, Jorge E. (2005). EL DESEMPLEO EN COLOMBIA Y SU METODOLOGÍA DE CÁLCULO ¿DISIMULO A UN GRAVE 


\section{8}

PROBLEMA?. Semestre Económico, 8(15),136150.[fecha de Consulta 17 de Marzo de 2021]. ISSN: 0120-6346. Disponible en: https://www. redalyc.org/articulo.oa?id=1650/165013659008

El Tiempo (2020). Contrato de arriendo, la sorpresa con la que Uber reta a Mintransporte. Recuperado de: https://n9.cl/e57o

Hernández Royett, J., Hernández, Y. F., Gil, M. de los A., \& Cárdenas Barboza, E. (2018). Evaluación del modelo integrado de planeación y gestión (MIPG) en las entidades territoriales del estado colombiano. Aglala, 9(1), 444-463. http:// revistas.curnvirtual.edu.co/index.php/aglala/ article/view/1255

Lesmes Silva, A. K., Barrientos-Monsalve, E. J., \& Cordero Díaz, M. C. (2020). Comunicación asertiva ¿estrategia de competitividad empresarial?. Aibi Revista De investigación, administración E ingeniería, 8(1), 147 - 153. https://doi.org/10.15649/2346030X.757

Monsalve, B. (2019). Theorization on case studies in business intelligence management on intellectual capital. Journal of Physics: Conference Series, 1160(1).

Narváez, B., Arrieta, Y. \& Flores, B. (2018). El caso del aplicativo móvil Uber frente al régimen de protección a la competencia en la legislación colombiana. Revista Justicia, 23(33), 37-50. https://doi.org/10.17081/just.23.33.2881

Ossa Bocanegra, Camilo ErnEsto (2017). Economías colaborativas: regulación y competencia. Revista de Derecho Privado, (57),1-22.[fecha de Consulta 17 de Marzo de 2021]. ISSN: . Disponible en: https://www.redalyc.org/articulo. oa?id=3600/360055996008

Santaella Pérez, H (2020) Informe de Estudio Económico de las Zonas. Cámara de Comercio Cúcuta. Recuperado de: https://www.cccucuta.
org.co/media/Publicaciones/informe estudio_economico_de_la_zona_dic_2019. pdf

Vega-Ortega, C., Medina-Gómez, D., \& Duarte, D. (2018). Revisión de la seguridad vial en la Av. $3 \mathrm{E}$ entre las calles $2 \mathrm{~N}$ y $6 \mathrm{~N}$ en la ciudad de Cúcuta para medir los riesgos a los que están expuestos los usuarios. Sostenibilidad, Tecnología Y Humanismo, 9(1), 8-15. https:// doi.org/10.25213/2216-1872.55 